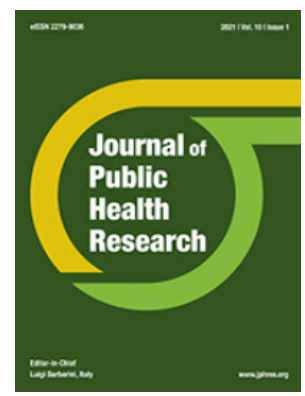

Journal of Public Health Research

elSSN 2279-9036

https://www.jphres.org/

Publisher's Disclaimer. E-publishing ahead of print is increasingly important for the rapid dissemination of science. The Journal of Public Health Research is, therefore, E-publishing PDF files of an early version of manuscripts that undergone a regular peer review and have been accepted for publication, but have not been through the copyediting, typesetting, pagination and proofreading processes, which may lead to differences between this version and the final one.

The final version of the manuscript will then appear on a regular issue of the journal. E-publishing of this PDF file has been approved by the authors.

J Public Health Res 2021 [Online ahead of print]

To cite this Article:

Ni B, Gettler E, Stern R, et al. Disruption of medical care among individuals in the southeastern United States during the COVID-19 pandemic. J Public Health Res doi: 10.4081/jphr.2021.2497

() (C) the Author(s), 2021

Licensee PAGEPress, Italy 


\title{
Disruption of medical care among individuals in the southeastern United States during the COVID-19
} pandemic

\author{
Bin Ni, ${ }^{1}$ Erin Gettler, ${ }^{1}$ Rebecca Stern, ${ }^{1}$ Heather M. Munro, ${ }^{2}$ Mark Steinwandel, ${ }^{2}$ Melinda C. Aldrich, ${ }^{3}$ \\ Debra L. Friedman, ${ }^{4}$ Maureen Sanderson, ${ }^{5}$ David Schlundt, ${ }^{6}$ David M. Aronoff, ${ }^{1}$ Deepak K. Gupta, ${ }^{7,8}$ \\ Martha J. Shrubsole, ${ }^{9}$ Loren Lipworth ${ }^{8,9}$
}

${ }^{1}$ Division of Infectious Diseases, Department of Medicine, Vanderbilt University Medical Center, Nashville, TN

${ }^{2}$ Vanderbilt Institute for Clinical and Translational Research, Vanderbilt University Medical Center, Nashville, TN

${ }^{3}$ Division of Genetic Medicine, Department of Medicine, Vanderbilt University Medical Center, Nashville, TN

${ }^{4}$ Division of Pediatric Hematology/Oncology, Department of Pediatrics, Vanderbilt University Medical Center, Nashville, TN

${ }^{5}$ Department of Family and Community Medicine, Meharry Medical College, Nashville, TN

${ }^{6}$ Department of Psychology, Vanderbilt University, Nashville, TN

${ }^{7}$ Division of Cardiovascular Medicine, Department of Medicine, Vanderbilt University Medical Center, Nashville, TN

${ }^{8}$ Vanderbilt Translational and Clinical Cardiovascular Research Center, Vanderbilt University Medical Center, Nashville, TN

${ }^{9}$ Division of Epidemiology, Department of Medicine, Vanderbilt University Medical Center, Nashville, TN, USA

Corresponding author: Loren Lipworth, Division of Epidemiology, Vanderbilt University Medical Center

2525 West End Ave, Suite 800, Nashville, TN 37203, USA. Tel. +001.615.343-0639. E-mail:

loren.lipworth@vumc.org

Funding: Research reported in this publication was supported by the National Cancer Institute of the National Institutes of Health under Award Number U01CA202979 (HMM, MS, DLF, MS, MJS, LL). The content is solely the responsibility of the authors and does not necessarily represent the official views of the National Institutes of Health. SCCS data collection was performed by the Survey and Biospecimen Shared Resource which is supported in part by the Vanderbilt-Ingram Cancer Center (P30 CA68485; DLF, MJS). Dr. Aldrich is additionally funded by Award Number 1U01CA253560 from the National Cancer Institute.

Contributions: All authors made substantial contributions to the conception and design of the study, the acquisition and analysis of the data, or drafted the work and critically revised it. All authors had final approval of the manuscript and agree to be accountable for all aspects of the work.

Conflict of interest:The authors declare no known conflicts of interest associated with this publication. 
Availability of data and materials: The data used to support the findings of this study are available from the corresponding author upon request.

Ethics approval and consent to participate: Study protocols were approved by institutional review boards at Vanderbilt University Medical Center and Meharry Medical College (Protocol \#010345). Written informed consent was obtained from participants.

Patient consent for publication: Not applicable

Informed consent: The manuscript does not contain any individual person's data in any form.

\section{Significance for Public Health}

Lapses in routine medical care for chronic conditions and preventative healthcare due to the COVID-19 pandemic may have long-term adverse public health effects. These effects may be exacerbated among low-income individuals in the southeastern US who already experience higher rates of chronic disease and poor health outcomes. This study finds that the main drivers of healthcare lapses during the pandemic originate from the healthcare system through provider-initiated cancellations and from individual perceptions regarding the risk of contracting and surviving COVID-19, rather than medical comorbidities. The findings further identify a need for outreach efforts to re-engage patients in chronic and preventative care, as well as characteristics of groups that will benefit from targeted populationbased interventions, including women.

\section{Abstract}

Background: Widespread disruptions of medical care to mitigate COVID-19 spread and reduce burden on healthcare systems may have deleterious public health consequences.

Design and Methods: To examine factors contributing to healthcare interruptions during the pandemic, we conducted a COVID-19 impact survey between 10/7-12/14/2020 among participants of the Southern Community Cohort Study, which primarily enrolled low-income individuals in 12 southeastern states from 2002-2009. COVID survey data were combined with baseline and follow-up data.

Results: Among 4,463 respondents, 40\% reported having missed/delayed a health appointment during the pandemic; the common reason was provider-initiated cancellation or delay (63\%). In a multivariable model, female sex was the strongest independent predictor of interrupted care, with odds ratio (OR) 1.63 (95\% confidence interval $[\mathrm{Cl}] 1.40-1.89$ ). Those with higher education (OR 1.27; 95\% $\mathrm{Cl} 1.05-1.54$ for college graduate vs $\leq$ high school) and household income (OR 1.47; 95\% $\mathrm{Cl} 1.16-1.86$ for $>\$ 50,000$ vs $<\$ 15,000)$ were at significantly increased odds of missing healthcare. Having greater perceived risk for acquiring (OR 1.42; 95\% Cl 1.17-1.72) or dying from COVID-19 (OR 1.25; 95\% Cl 1.04-1.51) also significantly increased odds of missed/delayed healthcare. Age was inversely associated with missed healthcare among men (OR for 5 -year increase in age $0.88 ; 95 \% \mathrm{Cl} 0.80-0.96$ ) but not women (OR 0.97; 
95\% Cl 0.91-1.04; $p$-interaction=0.04). Neither race/ethnicity nor comorbidities were associated with interrupted healthcare.

Conclusions: Disruptions to healthcare disproportionately affected women and were primarily driven by health system-initiated deferrals and individual perceptions of COVID-19 risk, rather than medical comorbidities or other traditional barriers to healthcare access.

Key words: healthcare disruption, COVID-19, preventative care, low income

\section{Introduction}

The COVID-19 pandemic has resulted in major shifts in patterns of healthcare consumption and delivery in the United States (US). Many early community-level interventions, such as restrictions on leaving the home and deferral of elective and non-emergent medical procedures by hospitals, were aimed at "flattening the curve" to slow the rate of transmission and reduce burden to healthcare systems. This resulted in cancellations or delays of many outpatient or routine healthcare appointments (1-3). Although Medicare expanded coverage for telemedicine, telehealth has only offset a fraction of the decrease in inperson healthcare visits (3-5) and many Americans continue to have lapses in chronic or preventive medical care resulting in negative health consequences (6-8). Deleterious effects of these disruptions are likely to be exacerbated in certain groups including older adults, minority populations, and those with socioeconomic disadvantage, who not only bear an unequal burden of COVID-19, but who are also disproportionately affected by high prevalence of chronic diseases and their risk factors $(9,10)$. Therefore, we examined sociodemographic characteristics, living circumstances, and health-related factors, including perceived risk of COVID-19 exposure, contributing to interruptions in planned healthcare during the pandemic among participants in the Southern Community Cohort Study (SCCS), a prospective cohort study that primarily enrolled low-income individuals in the southeast US.

\section{Design and Methods}

\section{Study design and data collection}

The SCCS enrolled approximately 86,000 English-speaking adults aged 40-79 years from 2002-2009 in 12 southeastern states. (11). Eighty-six percent of participants were enrolled through Community Health Centers $(\mathrm{CHC})$, which provide healthcare services to underserved populations, with the remaining $14 \%$ recruited via population sampling. Study protocols were approved by institutional review boards at 
Vanderbilt University Medical Center and Meharry Medical College. Written informed consent was obtained from participants.

Study participants completed a survey at cohort enrollment and up to four follow-up surveys to collect detailed information on sociodemographic, lifestyle, medical and other variables. This analysis incorporated responses from these prior surveys and, to assess the impact of the COVID-19 pandemic, we developed a new comprehensive survey with questions on SARS-CoV-2 infection, physical and emotional health, COVID-19 related behaviors and beliefs, and household and healthcare impacts. Survey questions were obtained or modified from various sources or developed by the research team (https://www.southerncommunitystudy.org/questionnaires.html). Participants were asked the following questions developed specifically to assess delay or avoidance of medical care during the pandemic (yes/no): a) 'Since March 1, 2020, did you ever miss a healthcare appointment (with a doctor, nurse practitioner, nurse, or dentist)?'; b) 'Since March 1, 2020, was any healthcare procedure delayed (like a surgery, colonoscopy, or mammogram)?' Individuals who responded 'yes' were asked to select reasons for missed/delayed healthcare from a list of pre-specified responses.

\section{Survey Implementation}

Following a pilot of the survey, the fielding period for the full COVID-19 impact survey was 10/7/2020 to 12/14/2020. Participants were paid $\$ 10$ for completing the 20 -minute online self-administered survey. SCCS participants were notified about the survey through two pathways (see Supplemental Figure 1). Overall, 4,512 participants completed the survey, $98 \%$ of whom completed all questions. The American Association for Public Opinion Research Response Rate \#1 among participants emailed a direct invitation was $24.4 \%$.

\section{Statistical Analysis}

Analyses were based on 4,463 participants who provided complete information on the survey questions related to delayed medical care. We summarized participant characteristics by frequencies (percentages) or medians $\left(25^{\text {th }}, 75^{\text {th }}\right.$ percentiles), and used the Kruskal-Wallis and Pearson chi-squared tests to compare continuous and categorical variables, respectively. Multivariable logistic regression was performed to estimate odds ratios (OR) and 95\% confidence intervals (Cl) for associations between participant characteristics and avoidance or delay of medical care. We first assessed the association of each individual variable with disruption of care by adjusting for age (years, continuous), self-reported race/ethnicity (nonHispanic Black, non-Hispanic White, other/unknown), and sex in logistic regression models. We tested 
whether the association between age and delayed healthcare was non-linear, and the result was not statistically significant, therefore age was modeled as a linear variable. The variables we tested (defined as shown in Table 1) included: a) sociodemographic: household income (from the prior follow-up survey), education, health insurance status, current employment; b) health status: comorbidities, currently undergoing treatment for cancer, body mass index (BMI), general health status, number of clinic visits in the 12 months preceding the last follow-up survey, having a personal doctor, usual source of care; $c$ ) COVID-19-related: perceived likelihood of contracting COVID-19 or surviving COVID-19, change in household income during pandemic, change in employment during pandemic, SARS-CoV-2 infection testing and result; and d) household: urban-rural classification of residence using the 2013 Rural-Urban Continuum Codes for participants' geocoded county of residence, home type, lives alone. Those factors having a p-value $<0.05$ were then included as covariates in an age-, race/ethnicity-, and sex-adjusted multivariable model. Although having diabetes, hypertension, heart disease, kidney disease, or undergoing active cancer treatment were not significant in the individual models, they were retained in the full model because they were selected a priori as potential risk factors for disruption of care. If comorbidities or BMI were missing from the COVID-19 survey, we utilized the information provided on the most recent SCCS survey. We performed additional analyses to test whether the association between age, race/ethnicity or other covariates in the model and delayed healthcare was modified by sex. We calculated relative explained variation as a measure of the proportion of variation in the outcome explained by each grouping of variables in our multivariable model (e.g., sociodemographic, health status, and COVID-19-related)(12). Analyses were performed using SAS 9.4 and Stata 14.

\section{Results}

Descriptive characteristics of the 4,512 survey respondents are presented in Supplemental Table 1. The median age of respondents was 66 years, $66 \%$ were female, 38\% were Black, and $21 \%$ resided in rural areas. Overall, $22 \%$ had high school education or less, 57\% reported an annual household income below $\$ 50,000$, and $18 \%$ had income below $\$ 15,000$. Two-thirds of respondents utilized Medicaid or Medicare as primary insurance. Nearly all respondents were actively engaged in medical care prior to the pandemic; 93\% reported at least one healthcare visit in the previous 12 months. While $46 \%$ of respondents selfreported their general health status as very good or excellent, almost three-fourths had diabetes, hypertension, heart disease and/or kidney disease and $5 \%$ were actively undergoing cancer treatment. 
A missed or delayed healthcare appointment or procedure during the pandemic was reported by 1,765 of 4,463 respondents included in our analysis (39.5\%; Table 1). Those who missed appointments were younger, more likely to be female ( $73 \%$ vs. $61 \%)$, and more likely to have higher education, private health insurance, respiratory diseases or immunodeficiency diseases than those who did not miss appointments. $20 \%$ of individuals who missed appointments reported fair/poor general health status compared with $15 \%$ of those who did not miss appointments. Approximately $70 \%$ of respondents lived with other adults or children; a minority reported living with children under the age of 18 years ( $n=415)$ (data not shown). The proportion of respondents whose household income or work hours decreased during the pandemic was higher among those who missed appointments ( $26 \%$ and $23 \%$, respectively) than those who did not ( $21 \%$ and 19\%, respectively), as were both COVID-19 infection and perceived risk of COVID-19.

The most common reason for disruption to healthcare was that the provider postponed or cancelled the appointment $(1,112 / 1,765 ; 63 \%)$, followed by fear of COVID (34\%). A smaller number of respondents cited being too ill, lack of transportation, or concerns about paying for the visit (4-5\% for each) or "other" (17\%) as reasons for missing appointments (data not shown). When stratified by sex, fear of COVID (40\%) and being too ill (6\%) were reported significantly more frequently by women than men (32\% and $3 \%$, respectively) as reasons for missing healthcare. There was no significant difference by sex in the proportion citing cost concerns.

Approximately $54 \%$ of individuals who missed/delayed health visits reported receiving a telehealth visit (Table 1); $7 \%$ of those who did not receive telehealth reported wanting or being asked to have a telehealth visit but either not knowing how or not having equipment to do so. Among those who missed/delayed health visits, Black individuals were more likely to use telehealth than White individuals (60\% vs. $50 \%$ ), as were those with household income $<\$ 15,000(67 \%)$ compared to those with higher income levels $(50 \%$ for income $>\$ 50,000$ and $53 \%$ for income $\$ 15,000-\$ 49,999)$, while there were no apparent differences in telehealth use according to age or sex (data not shown). In the subset of 1,112 individuals whose appointment was cancelled or postponed by their provider, $59 \%$ had telehealth. Of the respondents with disrupted healthcare, $92 \%$ regularly used medical supplies or prescriptions; $7 \%$ of these individuals reported not having access to prescriptions or medical supplies due to the pandemic (data not shown).

Results of the multivariable logistic regression model are presented in Figure 1. Women were significantly more likely to experience interruptions in healthcare compared to men (OR 1.63, 95\% $\mathrm{Cl} 1.40-1.89$ ). Both 
household income and education level, but not race/ethnicity, were associated with interrupted healthcare. Individuals with household income $\$ 15,000-\$ 49,999$ or $\geq \$ 50,000$ had ORs of $1.38(95 \% \mathrm{Cl}$ 1.13-1.69) and 1.47 (95\% Cl 1.16-1.86), respectively, compared to those with income $<\$ 15,000$. Those with greater than high school education had approximately $25 \%$ greater odds of missing an appointment compared with individuals with $\leq$ high school education. Respondents with five or more healthcare visits in the previous 12 months had almost $60 \%$ greater odds (OR 1.57, 95\% Cl 1.32-1.87) of interrupted healthcare due to COVID-19. Participants who rated their general health as fair/poor were nonsignificantly more likely to miss appointments (OR 1.22, 95\% Cl 0.98-1.52) than excellent/very good health participants, as were those who had decreased household income during the past year (OR 1.19, 95\% Cl 0.99-1.43). With respect to other pandemic-related factors, previous testing for SARS-CoV-2, independent of the test result, was associated with a $20-50 \%$ increased odds of missing health appointments. ORs for missing appointments were $1.42(95 \% \mathrm{Cl} 1.17-1.72)$ among those with a perceived greater likelihood of contracting COVID-19 and 1.25 (95\% $\mathrm{Cl} 1.04-1.51)$ among those who deemed their probability of survival as unlikely if they were to acquire COVID-19. None of the other characteristics, including underlying comorbidities or active treatment for cancer, was significantly associated with disrupted healthcare. We observed a statistically significant interaction between sex and age $(p=0.04)$; age was inversely associated with odds of missing healthcare appointments among men (OR for 5 -year increase in age $0.88 ; 95 \% \mathrm{Cl}$ 0.80-0.96) but not among women (OR 0.97; 95\% Cl 0.91-1.04) (Figure 2).

Socioeconomic variables as a group explained approximately $39 \%$ of variation in the outcome, while the contribution of health status variables and pandemic variables were $24 \%$ and $20 \%$, respectively. Plots of predicted probabilities of missed/delayed healthcare for select patient characteristics (age, sex, general health status, and income level as one proxy for socioeconomic status) are presented in Figure 3; these variables were selected as they are likely to be readily available to clinical and public health professionals and can help prioritize resource allocation for re-engagement.

\section{Discussion}

We leveraged the SCCS infrastructure and extensive baseline and follow-up data to field a timely survey to investigate associations of sociodemographic, health, and COVID-related factors with disruptions in previously scheduled healthcare visits during the COVID pandemic. Among individuals largely recruited from CHCs in the southeast US, a group typically underrepresented in cohort studies, $40 \%$ of survey respondents reported having missed/delayed a scheduled health appointment due to COVID-19, which is 
higher than nearly one-third of the US adult population(1) and 1 in 5 Medicare beneficiaries $(13,14)$ who reported missed care in previous studies. In our study population, with high prevalence of chronic conditions and sociodemographic disadvantages, ongoing disruptions in routine healthcare are likely to lead to worse outcomes and may account for a disproportionate excess of non-COVID deaths during the pandemic(15-17).

Female sex was the strongest independent predictor of healthcare disruption. Similar findings of decreased healthcare utilization among women have been previously reported(1, 7). A potential driver of this association may be the logistical challenges of accessing healthcare while serving in a caregiver role, which is disproportionately undertaken by women $(1,18,19)$. Among our survey respondents, who had a median age of 66 years, only a small number lived with children under the age of 18 years. However, we were unable to identify individuals who were primary caregivers for adults living in their household or for grandchildren living elsewhere, nor did we directly assess disruptions in caregiving responsibility during the pandemic. A higher proportion of women have previously been shown to postpone or forgo health or preventive services due to costs compared to men(20). In our study, concerns about cost were reported with similarly low frequency $(<5 \%)$ by women and men as the reason for avoiding planned healthcare, and no statistically significant interaction was observed between income, pandemic-related change in income, or health status and sex. However, our findings concur with other studies demonstrating a disproportionate effect of the pandemic on hindering preventative health services for women(21), underscoring that additional factors impact care among vulnerable female populations during the pandemic, after accounting for factors such as income and insurance coverage.

While racial and ethnic minorities, as well as individuals of older age, lower socioeconomic status, and with certain underlying medical comorbidities, have been disproportionately affected by SARS-CoV-2 infection and severe COVID-19(22-24), these traditional COVID-19 risk factors were not independent deterrents to accessing care in our study. In fact, older men were less likely to miss or delay scheduled care than their younger counterparts, as were individuals with lower income and education levels. Previous studies have identified having insurance coverage as an important determinant of healthcare access and utilization(1,25), but we did not observe a relationship between insurance status/type and disrupted healthcare in the setting of the COVID-19 pandemic, again suggesting that factors traditionally associated with barriers to healthcare access may not necessarily influence access in the same ways during this pandemic(6). While financial consideration was not a major reason for delaying healthcare, it is 
unlikely that financial factors are entirely unrelated as individuals living in households experiencing decreased income or decreased work hours during the pandemic did tend towards more missed health appointments.

Individual perceived high risk of acquiring or having poor outcomes from COVID-19 had a strong independent effect on avoiding care. This is mirrored by the one-third of respondents who reported "fear of COVID" as the reason for delaying/missing appointments. Furthermore, COVID-19 testing, regardless of outcome, was associated with lapses in healthcare. This may be due in part to quarantine pending test results as well as isolation periods for those diagnosed with COVID-19. Similarly, higher utilizers of healthcare including those with greater number of clinic visits in the previous year and with self-reported fair/poor health were more likely to miss/delay care. We did not collect data on overall number of appointments during the pandemic and thus cannot rule out the possibility that a larger number of appointments may simply make it more likely that an individual would miss at least one. Reassuringly, disrupted care was not significantly associated with any particular group of medical comorbidities, or with current cancer treatment. However, the extent of impact from lapses in time-sensitive preventative care, including cancer screenings, are likely not yet realized and may disproportionately affect women, who have experienced decreased rates of breast and cervical cancer screenings in addition to decline in colonoscopy and lung cancer screening rates $(2,26-28)$.

Healthcare centers in the South and in rural areas report some of the lowest rates of telehealth visits and face a disproportionate number of implementation barriers(29). Telehealth utilization in our survey respondents was higher than rates previously reported(29), but did not make up for the number of missed/delayed appointments. The percentage of respondents who wished to use telehealth but could not due to lack of knowledge or equipment was low, but this is likely an underestimate since the survey was administered only online and thereby selects for individuals with internet access and technologic knowledge. While telehealth cannot serve as a surrogate for medical procedures or directly provide access to medications or medical supplies, expanded telehealth can certainly ease many of the barriers of access due to COVID-19.

A striking result in our analysis was that provider-initiated cancellation or delay was the most common reason for healthcare postponement. Early in the pandemic, the intention of physician deferrals was to decrease exposure, preserve healthcare resources, and reduce strain on healthcare systems $(5,30,31)$. 
Despite easing of restrictions, the number of health visits has not returned to pre-pandemic levels $(2,26$, 32), indicating that lapsed health visits have not been made up and ongoing healthcare deferrals continue. Although we lack data on the number of missed/delayed appointments and procedures that were rescheduled among our participants, any appointments that remain unscheduled would put patients at risk for becoming lost to follow-up and negative health consequences. Even delays in screening and chronic disease management can lead to more severe disease once care is resumed(8). Therefore, it is essential that clinics and providers implement outreach efforts to re-engage and reschedule high-risk patients and instill confidence in patients and providers that the healthcare system can be safely engaged with adherence to public health measures.

Our findings are subject to several limitations. The overall survey response rate was low and the distribution of respondents was skewed to a higher proportion of Whites and higher education and income levels compared to non-responders and to the parent SCCS cohort (Supplemental Table 1). Furthermore, only English-speaking participants were enrolled in the SCCS, limiting our ability to examine the impact of language barriers on maintaining healthcare or using telehealth. However, the survey population still captured a wide distribution of sociodemographic and other factors and included a substantial proportion of low income, rural and Black individuals and women across the southeastern US, underrepresented in other cohorts, who are likely to be at highest risk for pandemic effects on healthcare disruption. We were unable to quantify the total number, or distinguish the specific types, of missed/delayed health appointments or procedures, nor could we determine whether telehealth visits were in lieu of specific missed health appointments. Finally, we could not define adverse health consequences or impacts on medication adherence of any missed/delayed healthcare encounters. These questions provide opportunities for future work through planned follow-up surveys and claims data.

In a predominantly low-income cohort in the southeastern US, $40 \%$ of individuals reported a missed or delayed medical appointment during the COVID-19 pandemic. The majority of survey respondents were older than 65 years, retired and using public insurance, a frequently under-studied group for whom this study provides important data on healthcare utilization during the pandemic. The major reasons accounting for missed/delayed visits were healthcare system driven cancellations or postponements and individual perceptions regarding risk of contracting and surviving COVID-19, rather than medical comorbidities. Women were disproportionately impacted and should be prioritized for outreach efforts to make up for lapsed healthcare. 


\section{References}

1. Czeisler M, Marynak K, Clarke KEN, et al. Delay or Avoidance of Medical Care Because of COVID-19Related Concerns - United States, June 2020. MMWR Morb Mortal Wkly Rep 2020;69:1250-7.

2. Miller MJ, Xu L, Qin J, Hahn EE, et al. Impact of COVID-19 on cervical cancer screening rates among women aged 21-65 years in a large integrated health care system - Southern California, January 1September 30, 2019, and January 1-September 30, 2020. MMWR Morb Mortal Wkly Rep 2021;70:109-13.

3. Whaley CM, Pera MF, Cantor J, et al. Changes in health services use among commercially insured US populations during the COVID-19 pandemic. JAMA Netw Open 2020;3:e2024984.

4. Patel SY, Mehrotra A, Huskamp HA, et al. Trends in outpatient care delivery and telemedicine during the COVID-19 pandemic in the US. JAMA Intern Med 2021;181:388-91.

5. Baum A, Kaboli PJ, Schwartz MD. Reduced in-person and increased telehealth outpatient visits during the COVID-19 pandemic. Ann Intern Med 2021;174:129-31.

6. Findling MG, Blendon RJ, Benson JM. Delayed Care with harmful health consequences-Reported experiences from national surveys during coronavirus disease 2019. JAMA Health Forum 2020;1:e201463-e.

7. Hartnett KP, Kite-Powell A, DeVies J, et al. Impact of the COVID-19 pandemic on emergency department visits - United States, January 1, 2019-May 30, 2020. MMWR Morb Mortal Wkly Rep 2020;69:699-704.

8. Wright A, Salazar A, Mirica M, et al. The invisible epidemic: Neglected chronic disease management during COVID-19. J Gen Intern Med 2020;35:2816-7.

9. Behrman P, Fitzgibbon ML, Dulin A, et al. Society of behavioral medicine statement on COVID-19 and rural health. Transl Behav Med 2021;11:625-30.

10. Zelner J, Trangucci R, Naraharisetti R, Cao A, Malosh R, Broen K, et al. Racial disparities in coronavirus disease 2019 (COVID-19) mortality are driven by unequal infection risks. Clin Infect Dis 2020;72:e8895.

11. Signorello LB, Hargreaves MK, Blot WJ. The Southern Community Cohort Study: investigating health disparities. J Health Care Poor Underserved 2010;21:s26-37.

12. Harrell Jr FE. Regression modeling strategies, with applications to linear models, logistic and ordinal regression, and survival analysis. New York: Springer; 2015.

13. Centers for Medicare and Medicaid Services. Medicare Current Beneficiary Survey Summer COVID19 Data Snapshot. 2020. Available from: https://www.cms.gov/medicare-current-beneficiary-surveysummer-2020-covid-19-data-snapshot

14. Centers for Medicare and Medicaid Services. Medicare Current Beneficiary Survey Fall 2020 COVID19 Data Snapshot. 2020. Available from: https://www.cms.gov/medicare-current-beneficiary-surveyfall-2020-covid-19-data-snapshot

15. Kim D, Lee $\mathrm{Y}$, Thorsness $\mathrm{R}$, Nguyen $\mathrm{KH}$, et al. Racial and ethnic disparities in excess deaths among persons with kidney failure during the COVID-19 pandemic, March-July 2020. Am J Kidney Dis 2021;77:827-9.

16. Rossen LM, Branum AM, Ahmad FB, Sutton P, Anderson RN. Excess deaths associated with COVID-19, by age and race and ethnicity - United States, January 26-October 3, 2020. MMWR Morb Mortal Wkly Rep 2020;69:1522-7.

17. Faust JS, Lin Z, del Rio C. Comparison of estimated excess deaths in New York City during the COVID19 and 1918 Influenza pandemics. JAMA Network Open 2020;3:e2017527.

18. Connor J, Madhavan S, Mokashi M, et al. Health risks and outcomes that disproportionately affect women during the Covid-19 pandemic: A review. Soc Sci Med 2020;266:113364.

19. Gausman J, Langer A. Sex and gender disparities in the COVID-19 pandemic. J Womens Health (Larchmt) 2020;29:465-6. 
20. Kaiser Family Foundation [Internet]. Women's coverage, access, and affordability: Key findings from the 2017 Kaiser Women's Health Survey. 2018. Available from: https://www.kff.org/womens-healthpolicy/issue-brief/womens-coverage-access-and-affordability-key-findings-from-the-2017-kaiserwomens-health-survey/

21. Kaiser Family Foundation [Internet]. Women's experiences with health care during the COVID-19 pandemic. Findings from the KFF Women's Health Survey. 2021. Available from: https://www.kff.org/womens-health-policy/issue-brief/womens-experiences-with-health-careduring-the-covid-19-pandemic-findings-from-the-kff-womens-health-survey/

22. Tai DBG, Shah A, Doubeni CA, et al. The disproportionate impact of COVID-19 on racial and ethnic minorities in the United States. Clin Infect Dis 2021;72:703-6.

23. Mackey K, Ayers CK, Kondo KK, et al. Racial and ethnic disparities in COVID-19-related infections, hospitalizations, and deaths: A Systematic review. Ann Intern Med 2021;174-362-73.

24. Garg S, Kim L, Whitaker M, et al. Hospitalization rates and characteristics of patients hospitalized with laboratory-confirmed coronavirus disease 2019 - COVID-NET, 14 states, March 1-30, 2020. MMWR Morb Mortal Wkly Rep 2020;69:458-64.

25. Sommers BD, Gawande AA, Baicker K. Health insurance coverage and health - What the recent evidence tells us. N Engl J Med 2017;377:586-93.

26. Patt D, Gordan L, Diaz M, et al. Impact of COVID-19 on cancer care: How the pandemic is delaying cancer diagnosis and treatment for American seniors. JCO Clin Cancer Inform 2020;4:1059-71.

27. Mitchell EP. Declines in cancer screening during COVID-19 pandemic. J Natl Med Assoc 2020;112:5634.

28. Sprague BL, Lowry KP, Miglioretti DL, et al. Changes in mammography utilization by women's characteristics during the first 5 months of the COVID-19 pandemic. J Natl Cancer Inst 2021;113:11617.

29. Demeke HB, Merali S, Marks S, et al. Trends in use of telehealth among health centers during the COVID-19 pandemic - United States, June 26-November 6, 2020. MMWR Morb Mortal Wkly Rep 2021;70:240-4.

30. Warner MA. Stop doing needless things! Saving Healthcare resources during COVID-19 and beyond. J Gen Intern Med 2020;35:2186-8.

31. Gatto M, Bertuzzo E, Mari L, et al. Spread and dynamics of the COVID-19 epidemic in Italy: Effects of emergency containment measures. Proc Natl Acad Sci USA 2020;117:10484-91.

32. Commonwealth Fund [Internet]. The impact of COVID-19 on outpatient visits in 2020: Visits Remained stable, despite a late surge in cases. 2020. Available from: https://www.commonwealthfund.org/publications/2021/feb/impact-covid-19-outpatient-visits2020-visits-stable-despite-late-surge 
Table 1. Characteristics of study participants according to reported disruption of medical care, Southern Community Cohort Study.

\begin{tabular}{|c|c|c|c|}
\hline & $\begin{array}{c}\text { Missed/Delayed } \\
\text { Health } \\
\text { Appointment } \\
\mathrm{N}=1,765\end{array}$ & $\begin{array}{c}\text { No } \\
\text { Missed/Delaye } \\
\text { d Health } \\
\text { Appointment } \\
\text { N=2,698 }\end{array}$ & $\begin{array}{c}\text { p- } \\
\text { value }^{\#}\end{array}$ \\
\hline \multicolumn{4}{|l|}{ SOCIODEMOGRAPHICS } \\
\hline \multicolumn{4}{|l|}{ Sex } \\
\hline Male & $479(27.1)$ & $1040(38.5)$ & $<0.0001$ \\
\hline Female & 1286 (72.9) & $1658(61.5)$ & \\
\hline \multicolumn{4}{|l|}{ Age at COVID-19 Survey (years) } \\
\hline$<65$ & $786(44.5)$ & 1056 (39.1) & $<0.0001$ \\
\hline $65-<75$ & $727(41.2)$ & $1147(42.5)$ & \\
\hline $75+$ & $252(14.3)$ & 495 (18.3) & \\
\hline Median $\left(25^{\text {th }}, 75^{\text {th }}\right.$ percentile $)$ & $66(61,72)$ & $67(62,73)$ & $<0.0001$ \\
\hline \multicolumn{4}{|l|}{ Race/Ethnicity } \\
\hline White, non-Hispanic & $947(53.7)$ & $1525(56.5)$ & 0.07 \\
\hline Black, non-Hispanic & $692(39.2)$ & 1016 (37.7) & \\
\hline Other or unknown $\S$ & $126(7.1)$ & $157(5.8)$ & \\
\hline \multicolumn{4}{|l|}{ Household income } \\
\hline$<\$ 15,000$ & $307(17.4)$ & 509 (18.9) & 0.27 \\
\hline$\$ 15,000-49,999$ & $697(39.5)$ & $1008(37.4)$ & \\
\hline$\$ 50,000+$ & $760(43.1)$ & $1178(43.7)$ & \\
\hline Missing & 1 & 3 & \\
\hline \multicolumn{4}{|l|}{ Education } \\
\hline$\leq \mathrm{HS}$ & $350(20.3)$ & $613(23.1)$ & 0.04 \\
\hline Some college/vocational training & $570(33.0)$ & $800(30.2)$ & \\
\hline College graduate or higher & $806(46.7)$ & $1238(46.7)$ & \\
\hline Missing & 39 & 47 & \\
\hline \multicolumn{4}{|l|}{ Current health insurance } \\
\hline Medicaid/Medicare & $1152(65.3)$ & $1807(67.2)$ & 0.02 \\
\hline Private & $458(26.0)$ & $601(22.3)$ & \\
\hline Military/Other & $101(5.7)$ & $193(7.2)$ & \\
\hline None & $53(3.0)$ & $89(3.3)$ & \\
\hline Missing & 1 & 8 & \\
\hline \multicolumn{4}{|l|}{ Current employment } \\
\hline Work full time & $390(22.1)$ & $618(22.9)$ & 0.78 \\
\hline Work part time & $156(8.8)$ & $229(8.5)$ & \\
\hline Unemployed & $80(4.5)$ & $109(4.0)$ & \\
\hline Retired/disability/homemaker & $1139(64.5)$ & $1738(64.5)$ & \\
\hline Missing & 0 & 4 & \\
\hline \multicolumn{4}{|l|}{ HEALTH STATUS } \\
\hline \multicolumn{4}{|c|}{ Diabetes, hypertension, heart disease, kidney disease } \\
\hline Yes & $1286(73.2)$ & $1957(72.8)$ & 0.76 \\
\hline No & $471(26.8)$ & $732(27.2)$ & \\
\hline Missing & 8 & 9 & \\
\hline \multicolumn{4}{|c|}{ Asthma, COPD, other chronic lung disease } \\
\hline Yes & $504(29.4)$ & $641(24.4)$ & 0.0002 \\
\hline No & $1208(70.6)$ & $1989(75.6)$ & \\
\hline Missing & 53 & 68 & \\
\hline
\end{tabular}




\begin{tabular}{|c|c|c|c|}
\hline & $\begin{array}{c}\text { Missed/Delayed } \\
\text { Health } \\
\text { Appointment } \\
\mathbf{N}=1,765 \\
\end{array}$ & $\begin{array}{c}\text { No } \\
\text { Missed/Delaye } \\
\text { d Health } \\
\text { Appointment } \\
\mathrm{N}=\mathbf{2 , 6 9 8} \\
\end{array}$ & $\begin{array}{c}\text { p- } \\
\text { value }^{\#}\end{array}$ \\
\hline \multicolumn{4}{|c|}{$\begin{array}{l}\text { Rheumatoid arthritis, lupus, HIV, other autoimmune } \\
\text { disorder }\end{array}$} \\
\hline Yes & $404(23.4)$ & $485(18.3)$ & $<0.0001$ \\
\hline No & $1320(76.6)$ & $2162(81.7)$ & \\
\hline Missing & 41 & 51 & \\
\hline \multicolumn{4}{|c|}{ Currently undergoing treatment for cancer } \\
\hline Yes & $98(5.6)$ & $137(5.1)$ & 0.49 \\
\hline No & $1663(94.4)$ & 2554 (94.9) & \\
\hline Missing & 4 & 7 & \\
\hline \multicolumn{4}{|l|}{ Body mass index, $\mathrm{kg} / \mathrm{m}^{2}$} \\
\hline Median $\left(25^{\text {th }}, 75^{\text {th }}\right.$ percentile $)$ & $28.9(25.0,34.5)$ & $\begin{array}{c}28.5(25.0 \\
33.2)\end{array}$ & 0.06 \\
\hline \multicolumn{4}{|l|}{ General Health Status } \\
\hline Fair/poor & $357(20.2)$ & $413(15.3)$ & $<0.0001$ \\
\hline Good & $657(37.2)$ & $961(35.6)$ & \\
\hline Very good/excellent & $750(42.5)$ & $1323(49.1)$ & \\
\hline Missing & 1 & 1 & \\
\hline \multicolumn{4}{|c|}{ Number of visits to clinic/doctor during previous 12 months } \\
\hline 0 & $102(5.8)$ & $219(8.2)$ & $<0.0001$ \\
\hline $1-2$ & $524(30.0)$ & $982(36.7)$ & \\
\hline $3-4$ & 495 (28.3) & $803(30.0)$ & \\
\hline 5 or more & $626(35.8)$ & $673(25.1)$ & \\
\hline Missing & 18 & 21 & \\
\hline \multicolumn{4}{|l|}{ Have personal doctor } \\
\hline Yes & $1593(92.2)$ & 2423 (91.7) & 0.50 \\
\hline No & $134(7.8)$ & $220(8.3)$ & \\
\hline Missing & 38 & 55 & \\
\hline \multicolumn{4}{|l|}{ Usual source of care } \\
\hline $\mathrm{CHC}$ or free clinic & $220(12.5)$ & 320 (11.9) & 0.64 \\
\hline Private doctor & 1339 (76.1) & $2078(77.3)$ & \\
\hline Other or no source & $201(11.4)$ & $290(10.8)$ & \\
\hline Missing & 5 & 10 & \\
\hline \multicolumn{4}{|l|}{ HOUSEHOLD } \\
\hline \multicolumn{4}{|l|}{ Rural or Urban } \\
\hline Rural & 343 (19.4) & $577(21.4)$ & 0.12 \\
\hline Urban & $1421(80.6)$ & $2121(78.6)$ & \\
\hline Missing & 1 & 0 & \\
\hline \multicolumn{4}{|l|}{ Home type } \\
\hline House & $1,344(76.2)$ & $2106(78.2)$ & 0.43 \\
\hline Apartment & $268(15.2)$ & $387(14.4)$ & \\
\hline Mobile home & $114(6.5)$ & $149(5.5)$ & \\
\hline Other & $37(2.1)$ & $52(1.9)$ & \\
\hline Missing & 2 & 4 & \\
\hline \multicolumn{4}{|l|}{ Household } \\
\hline Lives alone & $541(30.8)$ & 788 (29.3) & 0.29 \\
\hline Lives with other adults/children & $1218(69.2)$ & $1903(70.7)$ & \\
\hline Missing & 6 & 7 & \\
\hline COVID-19 PANDEMIC VARIABLES & & & \\
\hline
\end{tabular}




\begin{tabular}{|c|c|c|c|}
\hline & $\begin{array}{c}\text { Missed/Delayed } \\
\text { Health } \\
\text { Appointment } \\
\mathrm{N}=1,765 \\
\end{array}$ & $\begin{array}{c}\text { No } \\
\text { Missed/Delaye } \\
\text { d Health } \\
\text { Appointment } \\
\mathrm{N}=2,698 \\
\end{array}$ & $\begin{array}{c}\text { p- } \\
\text { value }^{\#}\end{array}$ \\
\hline \multicolumn{4}{|l|}{ Change in household income during pandemic } \\
\hline Income increased & $117(6.6)$ & $186(6.9)$ & 0.0001 \\
\hline Income decreased & $467(26.5)$ & $567(21.0)$ & \\
\hline Income stayed about the same & $1180(66.9)$ & $1941(72.0)$ & \\
\hline Missing & 1 & 4 & \\
\hline \multicolumn{4}{|l|}{ Change in employment during pandemic } \\
\hline $\begin{array}{l}\text { You or someone in your household lost job or had hours } \\
\text { reduced }\end{array}$ & $411(23.4)$ & 508 (18.9) & 0.0003 \\
\hline No one in household lost job or had hours reduced & $1345(76.6)$ & $2178(81.1)$ & \\
\hline Missing & 9 & 12 & \\
\hline \multicolumn{4}{|l|}{ SARS-CoV-2 test } \\
\hline Never had a SARS-CoV-2 test & $970(55.1)$ & $1672(62.2)$ & $<0.0001$ \\
\hline Was tested but never had positive SARS-CoV- 2 test & 727 (41.3) & $936(34.8)$ & \\
\hline Ever had positive SARS-CoV-2 test & $63(3.6)$ & 79 (2.9) & \\
\hline Missing & 5 & 11 & \\
\hline \multicolumn{4}{|l|}{$\begin{array}{l}\text { What do you think is your likelihood of getting a COVID-19 } \\
\text { infection? }\end{array}$} \\
\hline Very or somewhat unlikely & 845 (48.7) & $1500(56.1)$ & $<0.0001$ \\
\hline Neither unlikely nor likely & $523(30.2)$ & $753(28.2)$ & \\
\hline Very or somewhat likely & 303 (17.5) & $340(12.7)$ & \\
\hline Had COVID-19 & $63(3.6)$ & $79(3.0)$ & \\
\hline Missing & 31 & 26 & \\
\hline \multicolumn{4}{|l|}{$\begin{array}{l}\text { What do you think is the likelihood that you will survive } \\
\text { COVID-19 if you become infected? }\end{array}$} \\
\hline Very or somewhat unlikely & $348(20.2)$ & 439 (16.5) & 0.0008 \\
\hline Neither unlikely nor likely & $352(20.4)$ & 499 (18.8) & \\
\hline Very or somewhat likely & $964(55.8)$ & $1641(61.7)$ & \\
\hline Had COVID-19 & $63(3.6)$ & $79(3.0)$ & \\
\hline Missing & 38 & 40 & \\
\hline \multicolumn{4}{|l|}{ Had telehealth visit during COVID-19 pandemic } \\
\hline & & $1145(42.6)$ & $<0.000$ \\
\hline Yes & 949 (53.9) & & 1 \\
\hline No & $813(46.1)$ & $1544(57.4)$ & \\
\hline Missing & 3 & 9 & \\
\hline \multicolumn{4}{|l|}{$\begin{array}{l}\text { Desired or offered telehealth but unable to use due to lack } \\
\text { of knowledge or equipment }{ }^{\wedge}\end{array}$} \\
\hline Yes & $58(7.2)$ & $59(3.8)$ & 0.0004 \\
\hline No & $752(92.8)$ & $1478(96.2)$ & \\
\hline Missing & 3 & 7 & \\
\hline
\end{tabular}

'All values are displayed as $\mathrm{N}(\%)$, with the exception of age and BMI which are displayed as median ( $25^{\text {th }}$ percentile- $75^{\text {th }}$ percentile). \#Pvalue for the comparison by characteristic between those who did and did not avoid medical care (missing category excluded). ${ }^{\S}$ Other/unknown category includes Hispanic/Latino, Asian or Pacific Islander, American Indian or Alaska Native, other racial/ethnic groups, mixed race and participants with missing or unknown values. Among those who reported not having a telehealth visit during the pandemic 


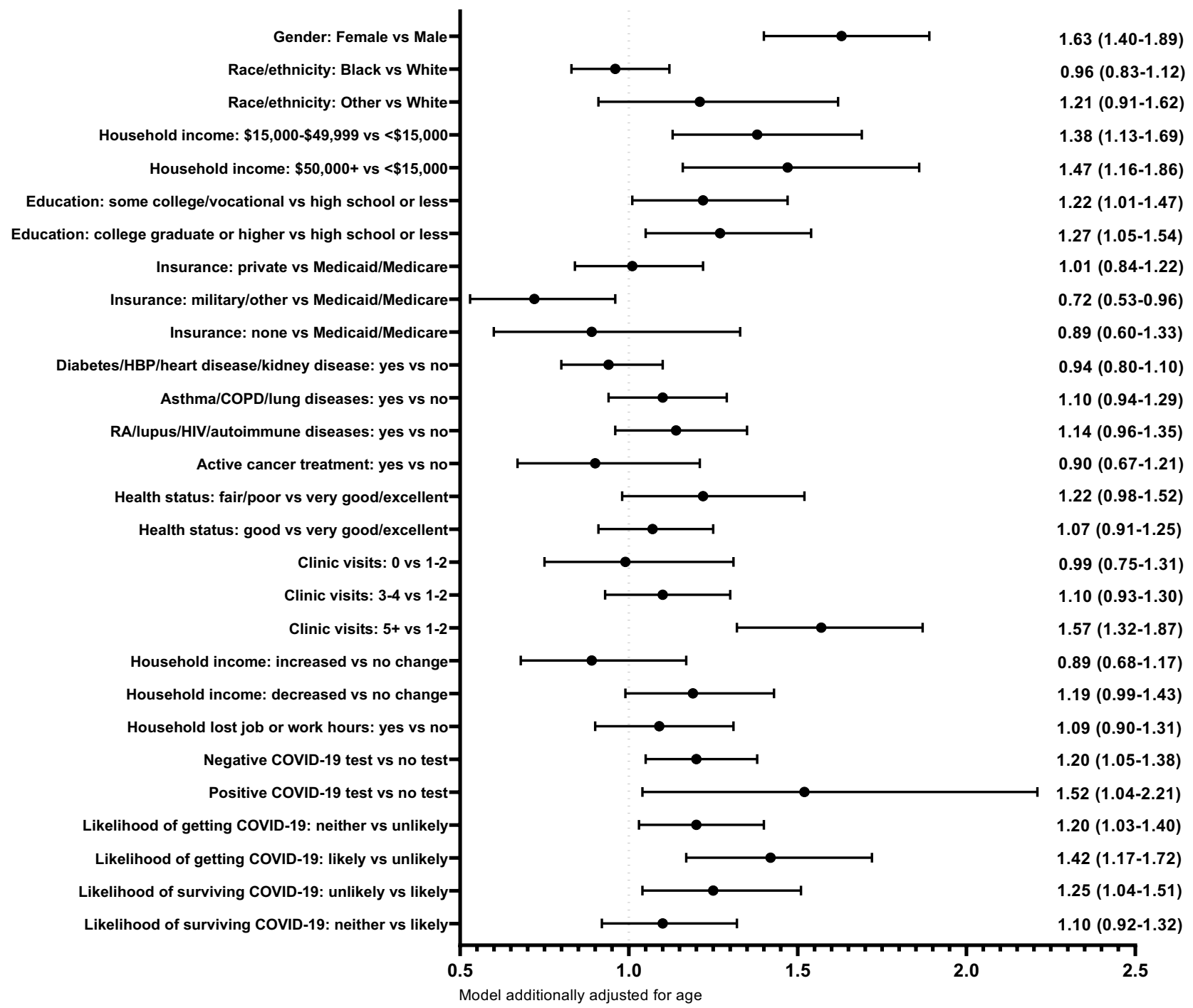

Figure 1. Multivariable-adjusted odds ratios and $95 \%$ confidence intervals for the association of participant characteristics with missed or delayed healthcare. Model additionally adjusted for age. 


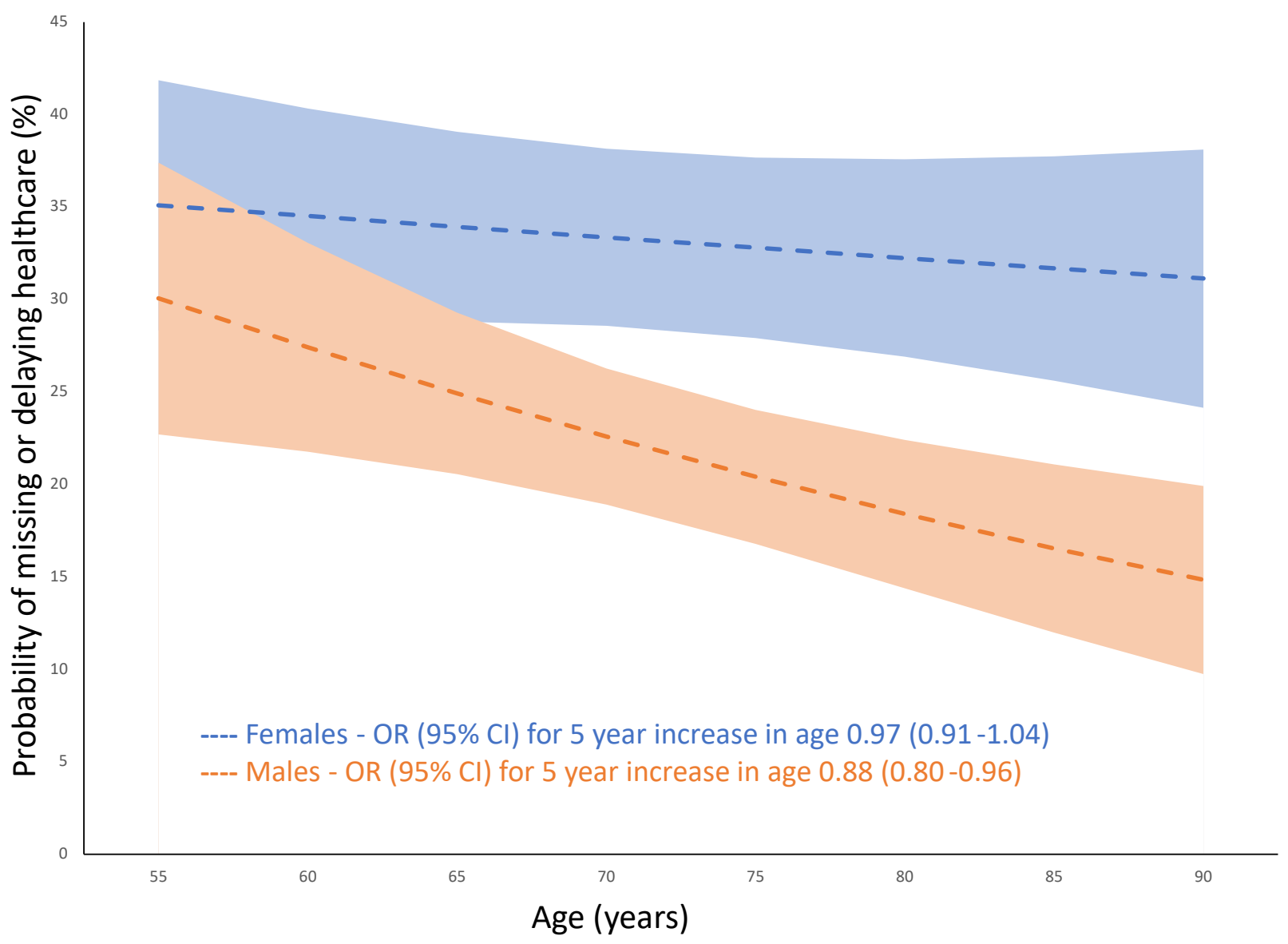

Figure 2. Differential association between age and missed healthcare appointments according to sex .

A statistically significant interaction between sex and age $(p=0.04)$ was observed. Age was inversely associated with odds of missing healthcare appointments among men but not among women. Other covariates in the multivariable model were assigned to their most common (mode) values of race/ethnicity (White), household income $(\$ 50,000+)$, education (college graduate or higher), insurance (Medicaid/Medicare), comorbidities (diabetes/high blood pressure/heart disease/kidney disease=yes; asthma/COPD/other chronic lung disease and rheumatoid arthritis/lupus/HIV/other autoimmune disorder=no), active cancer treatment (no), general health status (excellent/good), number of clinic visits (1-2), change in income during pandemic (no), change in employment during pandemic (no), COVID testing (not tested) and perceptions of risk of acquiring (unlikely) or surviving (likely) COVID-19. 

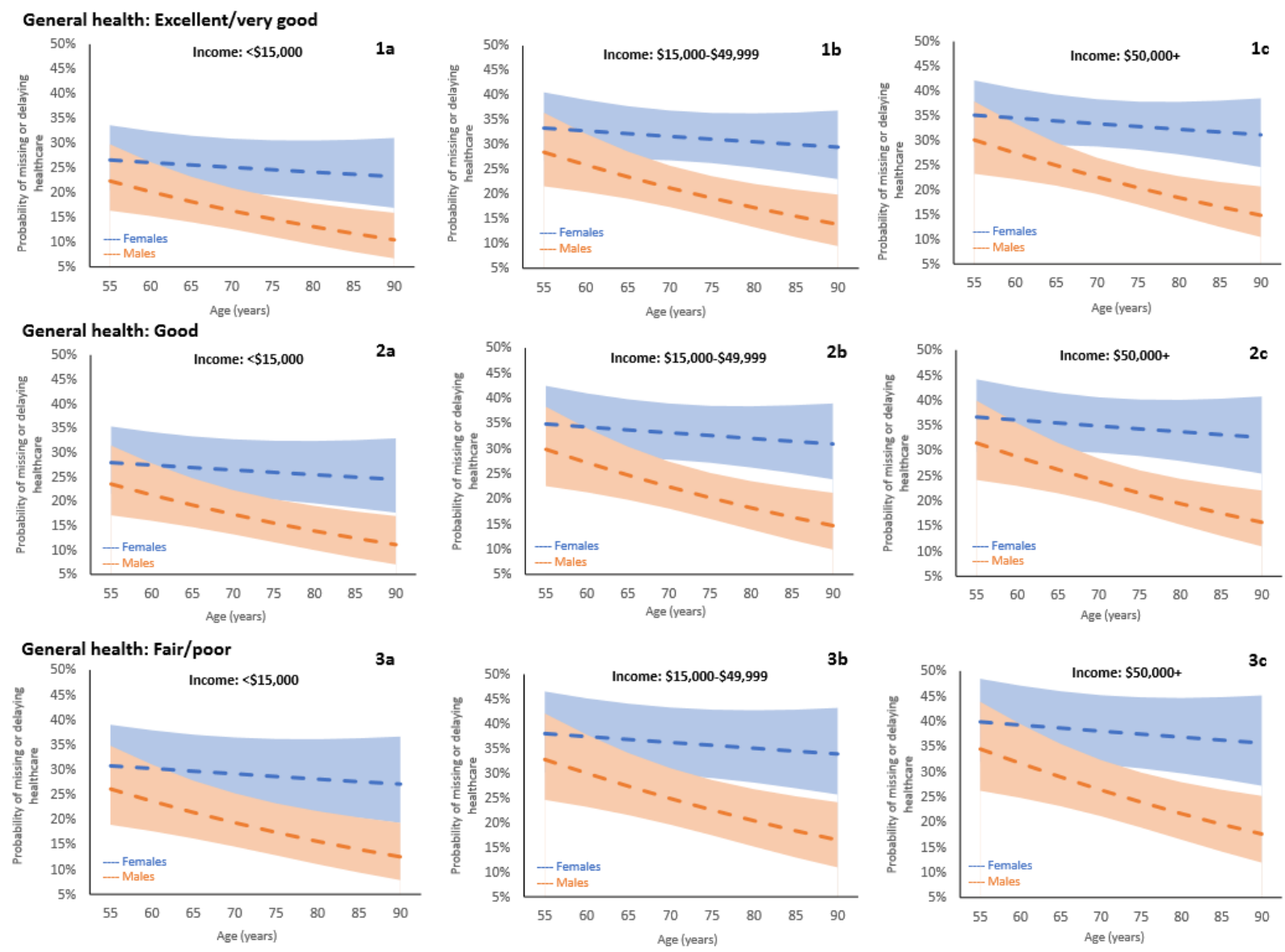

Figure 3. Predicted probabilities for missed or delayed healthcare by age, sex, general health status and income level. Predicted probabilities for missed or delayed healthcare according to age, sex and income level among those with excellent/very good general health status (1a-1c), good health status (2a-2c) or fair/poor health status (3a-3c). Other covariates in the multivariable model were assigned to their most common (mode) values of race/ethnicity, household income, education, insurance, comorbidities, active cancer treatment, general health status, number of clinic visits, change in income during pandemic, change in employment during pandemic, COVID testing and perceptions of risk of acquiring or surviving COVID-19 as listed in Figure 2. 